FACTA UNIVERSITATIS

Series: Mechanical Engineering Vol. 18, No 1, 2020, pp. 1 - 12

https://doi.org/10.22190/FUME200128011R

Original scientific paper

\title{
ELASTIC DAMPER BASED ON THE CARBON NANOTUBE BUNDLE
}

\author{
Leysan Kh. Rysaeva1, Elena A. Korznikova ${ }^{1,2}$, Ramil T. Murzaev ${ }^{1}$, \\ Dina U. Abdullina ${ }^{3}$, Aleksey A. Kudreyko ${ }^{4}$, Julia A. Baimova ${ }^{1}$, \\ Dmitry S. Lisovenko ${ }^{5}$, Sergey V. Dmitriev ${ }^{1,2}$ \\ ${ }^{1}$ Institute for Metals Superplasticity Problems of RAS, Russia \\ ${ }^{2}$ Institute for Molecule and Crystal Physics, UFRC, RAS, Russia \\ ${ }^{3}$ Bashkir State University, Russia \\ ${ }^{4}$ Department of Medical Physics and Informatics, Bashkir State Medical University, Russia \\ ${ }^{5}$ Ishlinsky Institute for Problems in Mechanics of the Russian Academy of Sciences, Russia
}

\begin{abstract}
Mechanical response of the carbon nanotube bundle to uniaxial and biaxial lateral compression followed by unloading is modeled under plane strain conditions. The chain model with a reduced number of degrees of freedom is employed with high efficiency. During loading, two critical values of strain are detected. Firstly, period doubling is observed as a result of the second order phase transition, and at higher compressive strain, the first order phase transition takes place when carbon nanotubes start to collapse. The loading-unloading stress-strain curves exhibit a hysteresis loop and, upon unloading, the structure returns to its initial form with no residual strain. This behavior of the nanotube bundle can be employed for the design of an elastic damper.
\end{abstract}

Key Words: Carbon Nanotube Bundle, Plane Strain Conditions, Lateral Compression Elastic Damper

\section{INTRODUCTION}

Carbon nanotubes (CNTs) attract each other due to relatively weak van der Waals interactions and can create molecular crystals or CNT bundles [1-3]. CNT forests can be produced by various techniques [4-7]. They have excellent mechanical properties since individual CNTs possess very high tensile strength in the range from 11 to $63 \mathrm{GPa}$, tensile Young's modulus of 1.0 to $1.3 \mathrm{TPa}$, and high ultimate fracture strain of about $10 \%$ [8-11]. Moreover, CNTs are flexible, lightweight, good heat and electricity conductors, thus being

Received January 28, 2020 / Accepted March 02, 2020

Corresponding author: Sergey V. Dmitriev

Institute for Metals Superplasticity Problems of RAS, Khalturin St. 39, Ufa 450001; Institute for Molecule and

Crystal Physics, UFRC, RAS, Oktyabrya Av., 151, Ufa 450075, Russia

E-mail: dmitriev.sergey.v@gmail.com 
useful in a number of applications [12-15]. The most important mechanical applications of CNTs are high-strength ropes [2,16], fibers [17-21], polymer- and metal-matrix composites [22-24], solid lubricants [24,25], etc.

It should be pointed out that tension [2,16-21] and compression [26-33] of vertically aligned CNT brushes and forests have been extensively studied. Note that CNTs grow in vertical direction and compression of vertically aligned CNTs means that they are compressed along their axis, while compression of horizontally aligned CNTs means that they are compressed normal to their axis. On the other hand, lateral compression of isolated CNTs or CNT bundles [34-38] is of interest, but this loading scheme has received much less attention of the researchers. Horizontally aligned CNT bundles can be obtained from the vertically aligned CNT arrays by winding, drawing, micromechanical rolling, and shear pressing [39-42]. Mechanical properties of CNTs, evaluated in the experimental and computational studies, have been outlined in the review [43]. CNT bundles are linear elastic up to hydrostatic pressure of $1.5 \mathrm{GPa}$; the volume compressibility is $0.024 \mathrm{GPa}^{-1}$; the hydrostatic deformation of CNT lattice is reversible up to the pressure of $4 \mathrm{GPa}$ [44]. With the help of the X-ray diffraction and Raman scattering, it has been found that deformation of CNT bundles under non-hydrostatic pressure is reversible below $5 \mathrm{GPa}$ [45].

Computational studies greatly contribute to a better understanding of mechanical properties of CNT bundles. Mesoscopic modeling of transformation of a low-density vertically aligned CNT forest into a horizontally aligned CNT forest under pressure has been addressed in $[46,47]$. A continuum shell model has been used to describe different morphological patterns of CNTs subjected to large deformations [48]. Applicability of the continuum beam model and nonlocal beam, plate and shell theories to the modeling of the mechanical properties of nanomaterials has been discussed in [49,50]. With increasing CNT diameter, the rigidity of CNT crystal does not decrease [1]. If CNT diameter is above a threshold value, it can be found in two stable configurations, circular and collapsed ones [51-53]. According to the experimental and molecular dynamics investigation, triple-wall CNT bundles under pressure exhibit irreversible transformation in the range of pressure from 60 to $72 \mathrm{GPa}$, depending on the temperature [34]. Mechanical response and failure of CNT bundles can be efficiently studied with the help of the nonlinear coarse-grained potentials developed for CNTs [54]. The chain model has been developed in [55] in order to study structure and properties of secondary, van der Waals conformations of carbon nanoribbons, such as folds and scrolls [55-59] as well as dynamics of surface ripplocations [60]. The chain model has been adopted to the study of CNT bundles under lateral compression in plane strain conditions in the work [61].

Vibration and shock protection applications require development of the materials possessing properties of elasticity and damping. Such properties are demonstrated, for example, by rubber or porous metallic rubber $[62,63]$. Such analogs have been proposed for nanomaterials as well. The armchair graphene nanoribbons under tension, at certain value of tensile strain, split into domains with large and small elastic strain [64]. Nucleation and motion of domain walls separating such domains produces considerable dissipation of elastic strain energy, and the loading-unloading stress-strain curves demonstrate hysteresis loop [64]. Cyclic loading of suspended $\mathrm{MoS}_{2}$ sheets during the nanoindentation experiments has also revealed the hysteresis behavior [65]. Damping properties of the compressed vertically aligned CNT brushes have been analyzed in [26].

In the present work, damping properties of the laterally compressed CNT bundle are analyzed in frame of the model developed in [61]. 


\section{Simulation Details}

We consider the nanotube bundle with the zigzag CNTs of equal diameter aligned along the $z$-axis, see Fig. 1 . Indices $i=1, \ldots, I$ and $j=1, \ldots, J$ number the CNTs. In Fig. 1 the case of $I=J=2$ is shown, while in this work we take $I=10, J=12$. Atoms within each CNT are numbered by the index $n=1, \ldots, N$ anti-clockwise, starting with the most right atom, as shown by red symbols in Fig. 1. In this work, lateral compression of the CNT bundle is considered under plane strain conditions, meaning that each carbon atom stands for a rigid atomic row oriented along the $z$-axis, which moves as a rigid body on the $(x, y)$ plane. Each atom has two degrees of freedom, namely, the components of the displacement vector on the $(x, y)$ plane, thus the dimensionality of the problem is reduced from $3 \mathrm{D}$ to $2 \mathrm{D}$. Total number of atoms in our model is $I \times J \times N=3600$. Periodic boundary conditions are imposed in both directions.

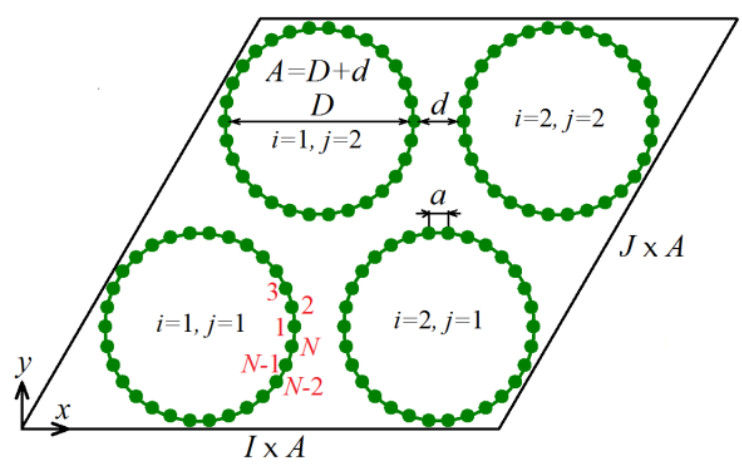

Fig. 1 Geometry of the computational cell, which includes $I \times J$ CNTs $(I=10, J=12$ in this work and $I=J=2$ in this figure) numbered by the indices $i=1, \ldots, I$ and $j=1, \ldots, J$, arranged in cross-section in a triangular lattice. Within each CNT atoms are numbered by the index $n=1, \ldots, N=30$ anti-clockwise, starting from the most right atom. Atoms have two degrees of freedom, the components of the displacement vector in the $(x, y)$ plane. Atomic chains normal to the $(x, y)$ plane move as rigid bodies. The computational cell size is defined by the distance between centers of neighboring CNTs, $A$, which is the sum of CNT diameter $D$ and distance between CNT walls $d$. Distance between atoms in the CNT wall is $a$. Periodic boundary conditions are imposed

The valence bond length in graphene is $\rho=1.418 \AA$. The distance between neighboring atomic rows in the zigzag CNT is $a=3^{1 / 2} \rho / 2=1.228 \AA$. CNT diameter is $D=a / \sin (\pi / N)$. The distance between CNT walls is $d$, then the centers of neighboring CNTs are at the distance $A=D+d$. The computational cell has the form of parallelogram with the sides $I \times A$ and $J \times A$. For the case of $N=30$ considered here, one has $D=11.75 \AA$ and equilibrium value of $d=3.088 \AA$. This choice of CNT diameter guarantees that an isolated CNT has single equilibrium state. If CNT diameter is greater than $\sim 3 \mathrm{~nm}$, then it has another equilibrium state, the collapsed one [51-53], and such CNTs may not open after unloading. The elastic damper discussed in this work operates due to CNT collapse during loading and opening during unloading. 
The Hamiltonian of the chain model employed in this study together with the model parameters can be found in [61]. It includes four terms,

$$
H=K+U_{B}+U_{A}+U_{V d W},
$$

where the first term in the right-hand side gives the kinetic energy of the carbon atoms, the second to the fourth terms stand for the energy of valence bonds, the energy of valence angles and the energy of van der Waals interactions between CNTs, respectively. The model parameters were calculated based on the Savin interatomic potential developed for $s p^{2}$-carbon [66] and successfully used for solving various problems [66-71].

Three strain-controlled loading schemes are considered: (i) uniaxial compression along close-packed CNT rows (i.e., along the $x$-axis with $\varepsilon_{x x}<0, \varepsilon_{y y}=0$ ), (ii) uniaxial compression normal to the close-packed CNT rows (i.e., along the $y$-axis with $\varepsilon_{x x}=0, \varepsilon_{y y}<0$ ), (iii) biaxial compression (with $\varepsilon_{x x}=\varepsilon_{y y}<0$ ). In all three cases compression up to certain level is followed by unloading to the zero strain. The strain state is characterized by the absolute value of volumetric strain $|\theta|=\left|\varepsilon_{x x}+\varepsilon_{y y}\right|$.

Perturbation-relaxation molecular dynamics at zero temperature is carried out aiming to obtain the equilibrium structures at different values of strain. The strain is applied by increments $\Delta \varepsilon_{i j}=-0.0025$ during compressive loading and $\Delta \varepsilon_{i j}=0.0025$ during unloading. Compression up to $|\theta|=0.3$ is analyzed for all three loading schemes, which is sufficient for the purpose of our study. Each strain increment is followed by perturbation of atomic positions with small random displacements along $x$ - and $y$-coordinates, uniformly distributed in the range from $-10^{-6}$ to $10^{-6} \AA$. Then the gradient method is applied to find the equilibrium structure by minimizing potential energy of the system. It is assumed that the energy has reached the minimal value when the absolute value of the maximal force acting on atoms becomes smaller than $10^{-10} \mathrm{eV} / \AA$.

\section{SIMULATION RESULTS}

Stress components $\sigma_{x x}$ and $\sigma_{y y}$ as the functions of the absolute value of the volumetric strain, $|\theta|=\left|\varepsilon_{x x}+\varepsilon_{y y}\right|$, are shown in Figs. 2 to 4 for the uniaxial compression along the $x$ axis, along the $y$-axis, and biaxial compression, respectively. Black (blue) lines are used for $\sigma_{x x}\left(\sigma_{y y}\right)$ stress, and solid lines are for loading, while dashed lines for unloading. Shear stress is not presented in the plots because it is either zero or, in some regimes, which will be discussed below, it is nonzero but still three orders of magnitude smaller than the normal stress components. Compression up to $|\theta|=0.3$ was simulated for all three loading schemes, but in the plots the most interesting range of strain, $|\theta| \leq 0.25$, was shown.

In Figs. 5 to 7, we present the evolution of the CNT bundle structure for the uniaxial compression along the $x$-axis, along the $y$-axis, and biaxial compression, respectively. The top rows stand for compression and the bottom rows for unloading. The absolute values of volumetric strain are given for each panel, and they correspond to the dots indicated in Figs. 2 to 4 . Note that the computational cell has the shape of a parallelogram but here, without loss of information, we present the structures in a rectangular widow using the fact that the cell is under periodic boundary conditions. 


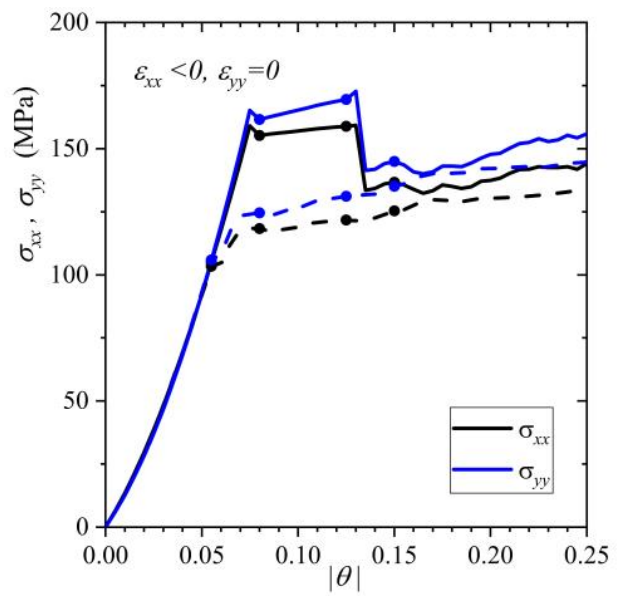

Fig. 2 Stress components as the functions of the absolute value of volumetric strain, $|\theta|=\left|\varepsilon_{x x}+\varepsilon_{y y}\right|$, for the case of uniaxial compression along the $x$-axis. Blue and black colors are used for $\sigma_{x x}$ and $\sigma_{y y}$, respectively. Loading and unloading is shown by solid and dashed lines, respectively. Dots indicate the values of elastic strain for which the structures are shown in Fig. 5

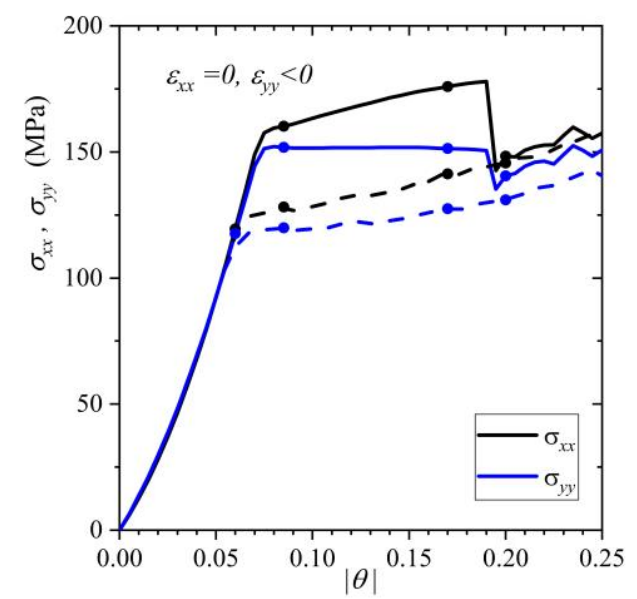

Fig. 3 Same as in Fig. 2, but for uniaxial compression along the $y$-axis. Dots indicate the values of elastic strain for which the structures are shown in Fig. 6 


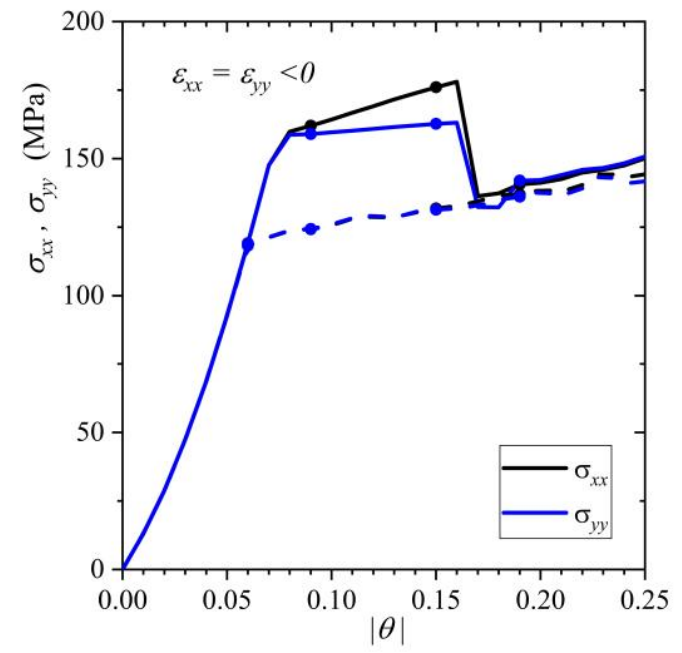

Fig. 4 Same as in Fig. 2, but for biaxial compression. Dots indicate the values of elastic strain for which the structure is shown in Fig. 7

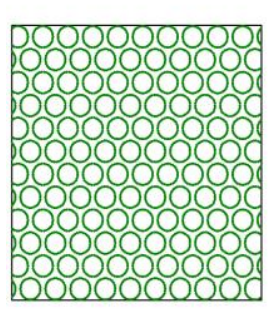

(a) $|\theta|=0.055$

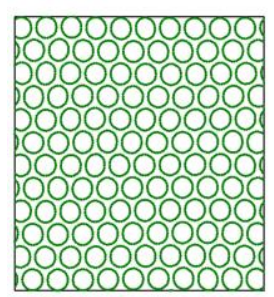

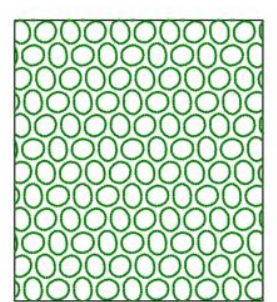

(b) $|\theta|=0.08$

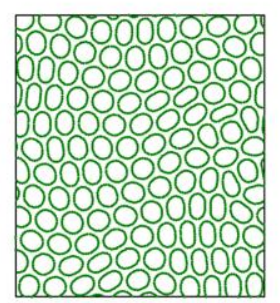

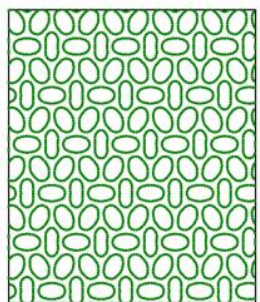

(c) $|\theta|=0.125$

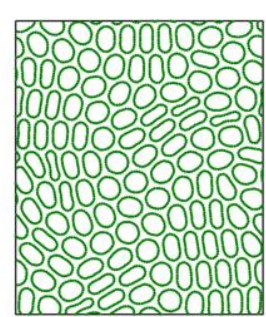

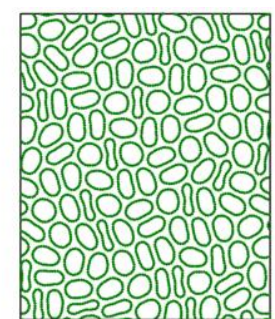

(d) $|\theta|=0.15$

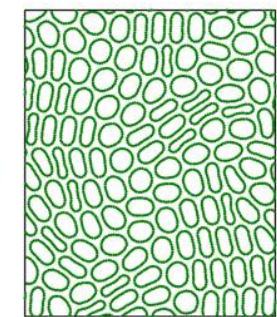

Fig. 5 Structure of the CNT bundle at different absolute values of volumetric strain, $|\theta|=\left|\varepsilon_{x x}+\varepsilon_{y y}\right|$, for the case of uniaxial compression along the $x$-axis. The top row is for compression and the bottom row for unloading. The values of strain are given for each panel, and they correspond to the dots indicated in Fig. 2 


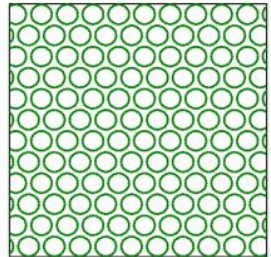

(a) $|\theta|=0.055$

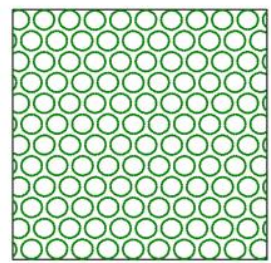

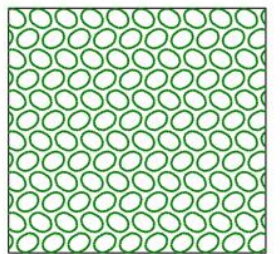

(b) $|\theta|=0.085$

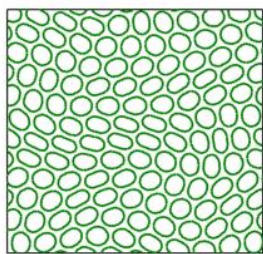

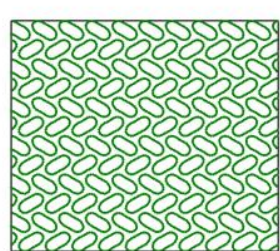

(c) $|\theta|=0.17$

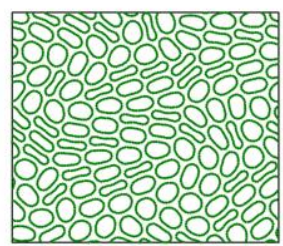

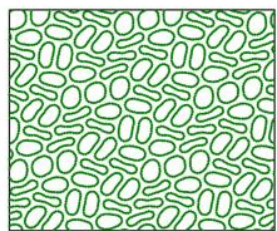

(d) $|\theta|=0.20$

Fig. 6 Same as in Fig. 5, but for the case of uniaxial compression along the $y$-axis. The values of strain are given for each panel, and they correspond to the dots indicated in Fig. 3

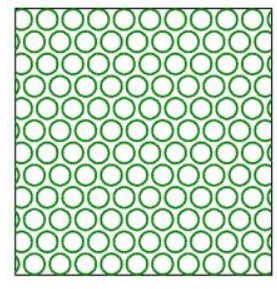

(a) $|\theta|=0.06$

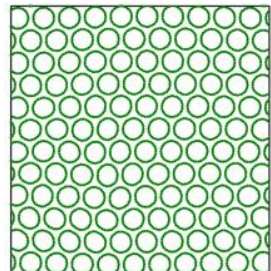

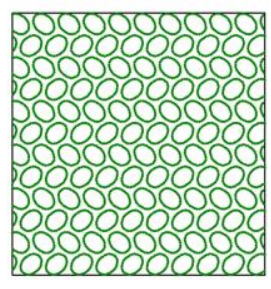

(b) $|\theta|=0.09$

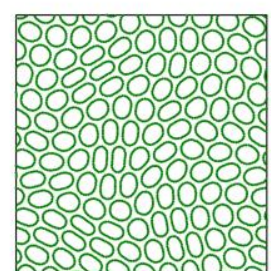

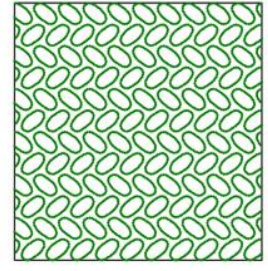

(c) $|\theta|=0.15$

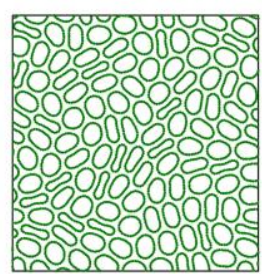

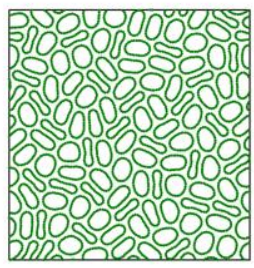

(d) $|\theta|=0.19$

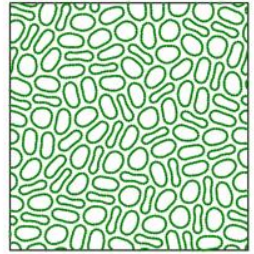

Fig. 7 Same as in Fig. 5, but for the case of biaxial compression. The values of strain are given for each panel, and they correspond to the dots indicated in Fig. 4

\section{DISCUSSION AND CONCLUSIONS}

The stress-strain curves, presented in Figs. 2 to 4, look similar for all three studied loading schemes, though some differences can be noticed. During compression from the initial state up to about $|\theta|=0.07$, the components of stress $\sigma_{x x}$ and $\sigma_{y y}$ are practically equal. This is expected for the case of biaxial loading due to the hexagonal symmetry of the structure, see Fig. 7(a), top row. On the other hand, this is quite unusual for uniaxial 
loading of an elastic body. The equality $\sigma_{x x}=\sigma_{y y}$ means that, in this regime of deformation, the Poisson's ratio of the CNT bundle under lateral compression is nearly equal to 1 , which is at the border of the thermodynamic stability for the two-dimensional elastic material [72,73]. As it can be seen in the panels (a) of Figs. 5 to 7, for $|\theta|<0.07$ the structure of the CNT bundle is very regular with all CNTs having the same slightly deformed cross section shape, and this is true for both loading (top row) and unloading (bottom row). Primitive translational cell of the CNT crystals in this regime includes single CNT.

When, on loading, the value of strain exceeds about $7 \%$ threshold, structural changes start to happen and the equality between components of normal stress is destroyed due to occurrence of the second-order phase transition. For compression along the $x$-axis, period doubling in both directions results in the primitive translational cell that includes four CNTs, see Fig. 5(b) and (c), top row. For compression along the $y$-axis and for biaxial compression, period doubling in one direction takes place and the primitive translational cell includes two CNTs, see Fig. 6(b) and (c), top row, as well as Fig. 7(b) and (c), top row. Both these structures have been described in [61]. In this regime all CNTs have elliptic, non-collapsed cross section for all three loading schemes. As for the normal stress components in this regime, one has $\sigma_{x x}<\sigma_{y y}$ for compression along the $x$-axis (see Fig. 2) and the opposite is true for compression along the $y$-axis and for biaxial compression (see Figs. 3 and 4).

On loading, appearance of CNTs with collapsed cross section is accompanied by a sudden drop of the normal stress components (first order phase transition). This drop takes place at $|\theta|=0.13,0.19$, and 0.16 in the case of compression along the $x$-axis, the $y$-axis, and biaxial compression, respectively, see Figs. 2 to 4 . Further compression after the drop of stress results in increase of stress and increase of the portion of collapsed CNTs. Structures with collapsed CNTs that appeared on loading can be seen in Figs. 5(d) to 7(d), top row.

During unloading from $|\theta|=0.30$, portion of the collapsed CNTs gradually decreases but they can be seen in bottom row even at $|\theta|=0.125$ in Fig. 5(c), at $|\theta|=0.17$ in Fig. 6(c), and at $|\theta|=0.15$ in Fig. 7(c), while in the corresponding top panels all CNTs have non-collapsed cross section. In the bottom rows of Figs. 5(b) to 7(b) all CNTs have non-collapsed elliptic cross section but the structures remain irregular, while corresponding top panels show regular structures. During unloading, at about $|\theta|=0.06$, for all three loading schemes, structure of the CNT bundles becomes regular, so that complete unloading results in complete restoration of the initial structure.

Structure difference observed in certain strain interval for the same values of strain on loading and unloading is the reason of the hysteresis loops that can be seen in Figs. 2 to 4. The area of the loop gives the energy dissipated by the structure during one loadingunloading cycle. This energy is maximal for the case of compression along the $y$-axis and minimal for loading along the $x$-axis.

We can conclude that the CNT bundle under lateral compression acts as a perfectly elastic damper. The efficiency of the energy absorption is highest when CNT bundle is compressed normal to the close-packed rows and it is intermediate for biaxial compression.

Practical design of elastic dampers based on CNT bundles requires further research [74]. In particular, the effect of CNT diameter is important to analyze. Also in many cases bundles of multi-walled CNTs are produced. It is tempting to investigate if they are more efficient absorbers of energy as compared to single-walled CNTs. These problems will be the topics of the future studies. Study of novel graphene-analogous 2D nanomaterials [7579] is also of high importance for the development of nano-sized dampers. 
Acknowledgements: This work was supported by the Russian Foundation for Basic Research, grant No. 18-32-20158. The work was partly supported by the State Assignment of IMSP RAS No. AAAA-A17-117041310220-8.

\section{REFERENCES}

1. Tersoff, J., Ruoff, R.S., 1994, Structural properties of a carbon-nanotube crystal, Phys. Rev. Lett., 73, pp. 676-679.

2. Thess, A., Lee, R., Nikolaev, P., Dai, H., Petit, P., Robert, J., Xu, C., Lee, Y.H., Kim, S.G., Rinzler, A.G., Colbert, D.T., Scuseria, G.E., Tomanek, D., Fischer, J.E., Smalley, R.E., 1996, Crystalline ropes of metallic carbon nanotubes, Science, 273, pp. 483-487.

3. Saether, E., Frankland, S.J.V., Pipes, R.B., 2003, Transverse mechanical properties of single-walled carbon nanotube crystals. Part I: Determination of elastic moduli, Compos. Sci. Technol., 63, pp. 1543-1550.

4. Rakov, E.G., 2013, Materials made of carbon nanotubes. The carbon nanotube forest, Russ. Chem. Rev., 82, pp. 538-566.

5. Chen, H., Roy, A., Baek, J.-B., Zhu, L., Qu, J., Dai, L., 2010, Controlled growth and modification of vertically-aligned carbon nanotubes for multifunctional applications, Mater. Sci. Eng. R: Reports, 70, pp. 63-91.

6. Bedewy, M., Meshot, E.R., Guo, H., Verploegen, E.A., Lu, W., Hart, A.J., 2009, Collective mechanism for the evolution and self-termination of vertically aligned carbon nanotube growth, J. Phys. Chem. C, 113, pp. 20576-20582.

7. Lan, Y., Wang, Y., Ren, Z.F., Physics and applications of aligned carbon nanotubes, Adv. Phys., 60, pp. 553-678.

8. Samsonidze, G.G., Samsonidze, G.G., Yakobson, B.I., 2002, Kinetic theory of symmetry-dependent strength in carbon nanotubes, Phys. Rev. Lett., 88, 065501.

9. Shenderova, O.A., Zhirnov, V.V., Brenner, D.W., 2002, Carbon nanostructures, Crit. Rev. Solid State, 27, pp. 227-356.

10. Yu, M.-F., 2004, Fundamental mechanical properties of carbon nanotubes: Current understanding and the related experimental studies, J. Eng. Mater. T. ASME, 126, pp. 271-278.

11. Yu, M.-F., Lourie, O., Dyer, M.J., Moloni, K., Kelly, T.F., Ruoff, R.S., 2000, Strength and breaking mechanism of multiwalled carbon nanotubes under tensile load, Science, 287, pp. 637-640.

12. Truong, T.K., Lee, Y., Suh, D., 2016, Multifunctional characterization of carbon nanotube sheets, yarns, and their composites, Curr. Appl. Phys., 16, pp. 1250-1258.

13. Yao, S., Yuan, J., Mehedi, H.-A., Gheeraert, E., Sylvestre, A., 2017, Carbon nanotube forest based electrostatic capacitor with excellent dielectric performances, Carbon, 116, pp. 648-654

14. Yao, X., Hawkins, S.C., Falzon, B.G., 2018, An advanced anti-icing/de-icing system utilizing highly aligned carbon nanotube webs, Carbon, 136, pp. 130-138.

15. Yao, X., Falzon, B.G., Hawkins, S.C., Tsantzalis, S., 2018, Aligned carbon nanotube webs embedded in a composite laminate: A route towards a highly tunable electro-thermal system, Carbon, 129, pp. 486-494.

16. Yu, M.-F., Files, B.S., Arepalli, S., Ruoff, R.S., 2000, Tensile loading of ropes of single wall carbon nanotubes and their mechanical properties, Phys. Rev. Lett., 84, pp. 5552-5555.

17. Dhanabalan, S.C., Dhanabalan, B., Chen, X., Ponraj, J.S., Zhang, H., 2019, Hybrid carbon nanostructured fibers: Stepping stone for intelligent textile-based electronics, Nanoscale, 11, pp. 3046-3101.

18. Bai, Y.,, Zhang, R., Ye, X., Zhu, Z., Xie, H., Shen, B., Cai, D., Liu, B., Zhang, C., Jia, Z., Zhang, S., Li, X., Wei, F., 2018, Carbon nanotube bundles with tensile strength over 80 GPa, Nat. Nanotechnol., 13, pp. 589-595.

19. Qiu, L., Wang, X., Tang, D., Zheng, X., Norris, P.M., Wen, D., Zhao, J., Zhang, X., Li, Q., 2016, Functionalization and densification of inter-bundle interfaces for improvement in electrical and thermal transport of carbon nanotube fibers, Carbon, 105, pp. 248-259.

20. Cho, H., Lee, H., Oh, E., Lee, S.-H., Park, J., Park, H.J., Yoon, S.-B., Lee, C.-H., Kwak, G.-H., Lee, W.J., Kim, J., Kim, J.E., Lee, K.-H., 2018, Hierarchical structure of carbon nanotube fibers, and the change of structure during densification by wet stretching, Carbon, 136, pp. 409-416.

21. Fernández-Toribio, J.C., Alemán, B., Ridruejo, Á., Vilatela, J.J., 2018, Tensile properties of carbon nanotube fibres described by the fibrillar crystallite model, Carbon, 133, pp. 44-52. 
22. Dang, Z.-M., Yuan, J.-K., Zha, J.-W., Zhou, T., Li, S.-T., Hu, G.-H., 2012, Fundamentals, processes and applications of high-permittivity polymer-matrix composites, Prog. Mater. Sci., 57, pp. 660-723.

23. Bakshi, S.R., Lahiri, D., Agarwal, A., 2010, Carbon nanotube reinforced metal matrix composites - A review, Int. Mater. Rev., 55, pp. 41-64.

24. Dorri Moghadam, A., Omrani, E., Menezes, P.L., Rohatgi, P.K., 2015, Mechanical and tribological properties of self-lubricating metal matrix nanocomposites reinforced by carbon nanotubes (CNTs) and graphene - A review, Compos. Part B: Eng., 77, pp. 402-420.

25. Reinert, L., Lasserre, F., Gachot, C., Grützmacher, P., Maclucas, T., Souza, N., Mücklich, F., Suarez, S., 2017, Long-lasting solid lubrication by CNT-coated patterned surfaces, Sci. Rep., 7, 42873.

26. Cao, A.Y., Dickrell, P.L., Sawyer, W.G., Ghasemi-Nejhad, M.N., Ajayan, P.M. 2005, Supercompressible foamlike carbon nanotube films, Science, 310, pp. 1307-1310.

27. Pathak, S., Kalidindi, S.R., 2015, Spherical nanoindentation stress-strain curves, Materials Science and Engineering R: Reports, 91, pp. 1-36.

28. Pathak, S., Cambaz, Z.G., Kalidindi, S.R., Swadener, J.G., Gogotsi, Y., 2009, Viscoelasticity and high buckling stress of dense carbon nanotube brushes, Carbon, 47, pp. 1969-1976.

29. Maschmann, M.R., Zhang, Q., Du, F., Dai, L., Baur, J., 2011, Length dependent foam-like mechanical response of axially indented vertically oriented carbon nanotube arrays. Carbon, 49, pp. 386-397.

30. Cao, C., Reiner, A., Chung, C., Chang, S.-H., Kao, I., Kukta, R.V., Korach, C.S., 2011, Buckling initiation and displacement dependence in compression of vertically aligned carbon nanotube arrays, Carbon, 49, pp. 3190-3199.

31. Liang, X., Shin, J., Magagnosc, D., Jiang, Y., Jin Park, S., John Hart, A., Turner, K., Gianola, D.S., Purohit, P.K., 2017, Compression and recovery of carbon nanotube forests described as a phase transition, Int. J. Solids Struct., 122-123, pp. 196-209.

32. Koumoulos, E.P., Charitidis, C.A., 2017, Surface analysis and mechanical behaviour mapping of vertically aligned CNT forest array through nanoindentation, Appl. Surf. Sci., 396, pp. 681-687.

33. Pour Shahid Saeed Abadi, P., Hutchens, S.B., Greer, J.R., Cola, B.A., Graham, S., 2013, Buckling-driven delamination of carbon nanotube forests, Appl. Phys. Lett., 102, 223103.

34. Silva-Santos, S.D., Alencar, R.S., Aguiar, A.L., Kim, Y.A., Muramatsu, H., Endo, M., Blanchard, N.P., San-Miguel, A., Souza Filho, A.G., 2019, From high pressure radial collapse to graphene ribbon formation in triple-wall carbon nanotubes, Carbon, 141, pp. 568-579.

35. Tangney, P., Capaz, R.B., Spataru, C.D., Cohen, M.L., Louie, S.G., 2005, Structural transformations of carbon nanotubes under hydrostatic pressure, Nano Lett., 5, pp. 2268-2273.

36. Zhang, S., Khare, R., Belytschko, T., Hsia, K.J., Mielke, S.L., Schatz, G.C., 2006, Transition states and minimum energy pathways for the collapse of carbon nanotubes, Phys. Rev. B, 73, 075423.

37. Shima, H., Sato, M., 2008, Multiple radial corrugations in multiwalled carbon nanotubes under pressure, Nanotechnology, 19, 495705

38. Zhao, Z.S., Zhou, X.-F., Hu, M., Yu, D.L., He, J.L., Wang, H.-T., Tian, Y.J., Xu, B., 2012, High-pressure behaviors of carbon nanotubes, J. Superhard Mater., 34, pp. 371-385.

39. Islam, S., Saleh, T., Asyraf, M.R.M., Mohamed Ali, M.S., 2019, An ex-situ method to convert vertically aligned carbon nanotubes array to horizontally aligned carbon nanotubes mat, Mater. Res. Express, 6 , 025019.

40. Zhang, R., Zhang, Y., Wei, F., 2017, Horizontally aligned carbon nanotube arrays: Growth mechanism, controlled synthesis, characterization, properties and applications, Chem. Soc. Rev., 46, pp. 3661-3715.

41. Nam, T.H., Goto, K., Yamaguchi, Y., Premalal, E.V.A., Shimamura, Y., Inoue, Y., Naito, K., Ogihara, S., 2015, Effects of CNT diameter on mechanical properties of aligned CNT sheets and composites, Compos. Part A: Appl. S., 76, pp. 289-298.

42. Qiu, L., Wang, X., Su, G., Tang, D., Zheng, X., Zhu, J., Wang, Z., Norris, P.M., Bradford, P.D., Zhu, Y., 2016, Remarkably enhanced thermal transport based on a flexible horizontally-aligned carbon nanotube array film, Sci. Rep., 6, 21014.

43. Qian, D., Wagner, G.J., Liu, W.K., Yu, M.-F., Ruoff, R.S., 2002, Mechanics of carbon nanotubes, Appl. Mech. Rev., 55, pp. 495-532.

44. Tang, J., Sasaki, T., Yudasaka, M., Matsushita, A., Iijima, S., 2000, Compressibility and polygonization of single-walled carbon nanotubes under hydrostatic pressure, Phys. Rev. Lett., 85, pp. 1887-1889.

45. Karmakar, S., Sharma, S.M., Teredesai, P.V., Muthu, D.V.S., Govindaraj, A., Sikka, S.K., Sood, A.K., 2003, Structural changes in single-walled carbon nanotubes under non-hydrostatic pressures: X-ray and Raman studies, New J. Phys., 5, pp. 143.1-143.11. 
46. Wittmaack, B.K., Volkov, A.N., Zhigilei, L.V., 2019, Phase transformation as the mechanism of mechanical deformation of vertically aligned carbon nanotube arrays: Insights from mesoscopic modeling, Carbon, 143, pp. 587-597.

47. Wittmaack, B.K., Volkov, A.N., Zhigilei, L.V., 2018, Mesoscopic modeling of the uniaxial compression and recovery of vertically aligned carbon, Compos. Sci. Technol., 166, pp. 66-85.

48. Yakobson, B.I., Brabec, C.J., Bernholc, J., 1996, Nanomechanics of carbon tubes: Instabilities beyond linear response, Phys. Rev. Lett., 76, pp. 2511-2514.

49. Rafii-Tabar, H., Ghavanloo, E., Fazelzadeh, S.A., 2016, Nonlocal continuum-based modeling of mechanical characteristics of nanoscopic structures, Phys. Rep., 638, pp. 1-97.

50. Harik, V.M., 2001, Ranges of applicability for the continuum beam model in the mechanics of carbon nanotubes and nanorods, Solid State Commun., 120, pp. 331-335.

51. Impellizzeri, A., Briddon, P., Ewels, C.P., 2019, Stacking- and chirality-dependent collapse of singlewalled carbon nanotubes: A large-scale density-functional study, Phys. Rev. B, 100, 115410.

52. Chopra, N.G., Benedict, L.X., Crespi, V.H., Cohen, M.L., Louie, S.G., Zettl, A., 1995, Fully collapsed carbon nanotubes, Nature, 377 , pp. 135-138.

53. Chang, T., 2008, Dominoes in carbon nanotubes, Phys. Rev. Lett., 101, 175501

54. Ji, J., Zhao, J., Guo, W., 2019, Novel nonlinear coarse-grained potentials of carbon nanotubes, J. Mech. Phys. Solids, 128, pp. 79-104.

55. Savin, A.V., Korznikova, E.A., Dmitriev, S.V., 2015, Scroll configurations of carbon nanoribbons, Phys. Rev. B, 92, 035412.

56. Savin, A.V., Korznikova, E.A., Dmitriev, S.V., 2015, Simulation of folded and scrolled packings of carbon nanoribbons, Phys. Solid State, 57, pp. 2348-2355.

57. Savin, A.V., Korznikova, E.A., Lobzenko, I.P., Baimova, Y.A., Dmitriev, S.V., 2016, Symmetric scrolled packings of multilayered carbon nanoribbons, Phys. Solid State, 58, pp. 1278-1284.

58. Savin, A.V., Korznikova, E.A., Dmitriev, S.V., Soboleva, E.G., 2017, Graphene nanoribbon winding around carbon nanotube, Comp. Mater. Sci., 135, pp. 99-108.

59. Savin, A.V., Mazo, M.A., 2019, 2D chain models of nanoribbon scrolls, Adv. Struct. Mat., 94, pp. 241-262.

60. Savin, A.V., Korznikova, E.A., Dmitriev, S.V., 2019, Dynamics of surface graphene ripplocations on a flat graphite substrate, Phys. Rev. B, 99, 235411.

61. Korznikova, E.A., Rysaeva, L.K., Savin, A.V., Soboleva, E.G., Ekomasov, E.G., Ilgamov, M.A., Dmitriev, S.V., 2019, Chain model for carbon nanotube bundle under plane strain conditions, Materials, 12(23), 3951.

62. Paimushin, V.N., Firsov, V.A., Gyunal, I., Shishkin, V.M., 2016, Identification of the elastic and damping characteristics of soft materials based on the analysis of damped flexural vibrations of test specimens, Mech. Compos. Mater., 52, pp. 435-454.

63. Ponomarev, Y.K., Ermakov, A.I., Simakov, O.B., Mikhalkin, I.K., 2013, Metallic counterpart of rubber: a material for vibration and shock protection, Met. Sci. Heat Treat., 55, pp. 8-13.

64. Evazzade, I., Lobzenko, I.P., Saadatmand, D., Korznikova, E.A., Zhou, K., Liu, B., Dmitriev, S.V., 2018, Graphene nanoribbon as an elastic damper, Nanotechnology, 29, 215704

65. Pang, H., Huang, P., Zhuo, W., Li, M., Gao, C., Guo, D., 2019, Hysteresis and its impact on characterization of mechanical properties of suspended monolayer molybdenum-disulfide sheets, Phys. Chem. Chem. Phys., 21, pp. 7454-7461.

66. Savin, A.V., Kivshar, Y.S., Hu, B., 2010, Suppression of thermal conductivity in graphene nanoribbons with rough edges, Phys. Rev. B, 82, 195422.

67. Savin, A.V., Korznikova, E.A., Krivtsov, A.M., Dmitriev, S.V., 2020, Longitudinal stiffness and thermal conductivity of twisted carbon nanoribbons, Eur. J. Mech. A-Solid., 80, 103920.

68. Savin, A.V., 2019, Thermal rectifiers based on asymmetric interaction of molecular chains, carbon nanoribbons, and nanotubes with thermostats, Phys. Rev. B, 10, 245415.

69. Shcherbinin, S.A., Semenova, M.N., Semenov, A.S., Korznikova, E.A., Chechin, G.M., Dmitriev, S.V., 2019, Dynamics of a three-component delocalized nonlinear vibrational mode in graphene, Phys. Solid State, 61, pp. 2139-2144.

70. Abdullina, D.U., Semenova, M.N., Semenov, A.S., Korznikova, E.A., Dmitriev, S.V., 2019, Stability of delocalized nonlinear vibrational modes in graphene lattice, Eur. Phys. J. B, 92, 249.

71. Savin, A.V., Korznikova, E.A., Dmitriev, S.V., 2019, Improving bending rigidity of graphene nanoribbons by twisting, Mech. Mater., 137, 103123.

72. Vasiliev, A.A., Dmitriev, S.V., Ishibashi, Y., Shigenari, T., 2002, Elastic properties of a two-dimensional model of crystals containing particles with rotational degrees of freedom, Phys. Rev. B, 65, 094101. 
73. Wojciechowski, K.W., 2003, Remarks on "Poisson ratio beyond the limits of the elasticity theory", J. Phys. Soc. Jpn, 72, pp. 1819-1820.

74. Prudkovskiy, V.S., Iacovella, F., Katin, K.P., Maslov, M.M., Cherkashin, N., 2018, A bottom-up approach for controlled deformation of carbon nanotubes through blistering of supporting substrate surface, Nanotechnology, 29, 365304.

75. Liu, B., Zhou, K., 2019, Recent progress on graphene-analogous $2 D$ nanomaterials: Properties, modeling and applications, Prog. Mater. Sci., 100, pp. 99-169.

76. Liu, B., Bai, L., Korznikova, E.A., Dmitriev, S.V., Law, A.W.-K., Zhou, K., 2017, Thermal conductivity and tensile response of phosphorene nanosheets with vacancy defects, J. Phys. Chem. C, 121, pp. 13876-13887.

77. Davletshin, A.R., Ustiuzhanina, S.V., Kistanov, A.A., Saadatmand, D., Dmitriev, S.V., Zhou, K., Korznikova, E.A., 2018, Electronic structure of graphene- and BN-supported phosphorene, Physica B, 534, pp. 63-67.

78. Bai, L., Srikanth, N., Korznikova, E.A., Baimova, J.A., Dmitriev, S.V., Zhou, K., 2017, Wear and friction between smooth or rough diamond-like carbon films and diamond tips, Wear, 372-373, pp. 12-20.

79. Baimova, J.A., Liu, B., Dmitriev, S.V., Srikanth, N., Zhou, K., 2014, Mechanical properties of bulk carbon nanostructures: Effect of loading and temperature, Phys. Chem. Chem. Phys. 16, pp. 1950519513. 